\title{
ROLE OF HAMSTRING MUSCLES IN KNEE JOINT STABILITY PROVIDING AND INJURY PREVENTION
}

\author{
Inese Pontaga \\ Academy of Sports Education, Latvia
}

\begin{abstract}
The aim of our investigation was to determine the ratio of maximal torque values and the torques in the certain positions of range of movements (ROM) between hamstring $(H)$ and quadriceps femoris $(Q)$ muscles at medium and high velocity of movementin concentric (CC) and eccentric (ECC) action of hamstring muscles.

The knee muscles of 15 amateur female short and middle distance runners were tested by the dynamometer system in the isokinetic movements with the angular velocity of $90 \%$ and $240 \% \mathrm{~s}$ in CC and at the velocity of $90 \%$ in ECC H/ CC Qmuscles contractions. The torque values produced by the muscles are detected at the different angular positions of the ROM with the step of $10^{\circ}$. The ratios of $H / Q$ muscles torques are calculated.

The $H / Q$ muscles maximal torques ratio is $0.51 \pm 0.13$ at the velocity of $90 \%$ in $C C$ and 0.60 \pm 0.09 in ECCH/ CC Q muscles contractions, and $0.59 \pm 0.09 \mathrm{CC}$ at the velocity of $240 \% \mathrm{~s}$. The H/Q maximal torques ratio and this ratio in the knee extreme extension and flexion at the ECC contraction of His higher due to greater torques produced by the $H$ in comparison with $Q$ muscle. The H must be stronger to decelerate the thigh and lower leg extension in the late swing phase of running and to extend the hip in early stance phase to provide powerful sprint running and prevent the knee and Hinjury. The H/Q muscles torques ratio in extended knee positions are similar in medium $(90 \%$ s) and fast $(240 \%$ s) velocity of motions because CC action of H muscles cannot prevent extreme knee extension.
\end{abstract}

Keywords: dynamometry, hamstring muscles, knee, muscles balance.

\section{Introduction}

Fast movements during running involve eccentric (ECC) and concentric (CC) contractions components, one following immediately after the other. This allows to reach higher force and power production and to provide smooth motions of the limbs. In the greatest part of the range of movements (ROM) the muscles contractions are CC. The ECC muscles action occurs only through few degrees of the ROM. Nosse (1982) andCoombs \& Garbutt (2002) think that it is not correct to determine the ratio of hamstrings $(\mathrm{H}) /$ quadriceps femoris $(\mathrm{Q})$ muscles maximal torques because they appear in the different positions (angles) of the knee joint ROM. Aagaard et al., $(1997,1998)$ have investigated an $\mathrm{ECCH} / \mathrm{CCQ}$ muscles contractions in the knee extension movement by is okinetic dynamometer and determined these muscles torques ratio $0.80-1.00$ for elite sailors and $0.80-0.84$ for male controls at the angle of ROM $50^{\circ}\left(0^{\circ}\right.$ - 
full knee extension). These ratios in solely $\mathrm{CC}$ are close to 0.50 . The $\mathrm{H} / \mathrm{Q}$ muscles torques ratios in all range of the knee joint movements in $\mathrm{CC}$ and $\mathrm{ECC}$ contractions of muscles and at different velocity of motions in the same athletes are not compared. These ratios values in the extreme positions of the ROM must be more informative in the joint stability estimation because a joint injury can occur more probable in these positions of the ROM. The aim of our investigation was to determine the ratio of maximal torque values and the torques in the certain positions of range of movements between knee flexor (hamstring) and extensor (m. quadriceps femoris) muscles at medium and high velocity of movement in concentric and eccentric action of hamstring muscles.

\section{Theoretical background}

Precise and smooth motions of the limbs depend on balanced action of muscles (agonists and antagonists) in the opposite sides of every joint. Weakness of one group of muscles can cause imbalanced motion in this joint leading to traumatization of musculoskeletal system due to incorrect distribution of the mechanical stresses in muscles, tendons, ligaments outside and inside the joint, and in the joint's surface cartilage. Only some investigations are available about a relationship between the knee flexor and extensor muscles strength balance and an occurrence of injuries in healthy athletes. For example, Knapik et al., (1991) revealed that the thigh muscles strength imbalance measured at fast velocity of movements by isokinetic dynamometer is associated with injuries. Another authors (Campbell \& Wayne, 1979; Kannus \& Jarvinen, 1990; Marshall $\&$ Tischler, 1978) determined that previous knee trauma and operation cause weakness of certain groups of muscles and imbalance of their action. Therefore repeated trauma often can occur. The predisposing factors to injury are (Campbell \& Wayne, 1979): changed muscles agonists/antagonist's strength, work and power balance, lack of flexibility in the joint, insufficient warmup before exercises and fatigue of muscles.

All three hamstring muscles are biarticular muscles: they are involved in hip extension and knee flexion, Fig. 1. During the late swing phase of sprint running or jumping the hamstrings work eccentrically to decelerate both the thigh and lower leg in preparation to ground contact (Marshall \& Tischler, 1978). Early in stance phase the hamstrings act concentrically to extent the hip. Kinetic investigations have shown that the maximal torques at the hip and knee occur during these phases (Whiting \& Zernicke, 1998).

If the $\mathrm{H}$ muscles are too weak in comparison with the $\mathrm{Q}$ muscle, it can change the muscles action balance and to create additional mechanical stresses on the knee anterior cruciate ligament (ACL) (Whiting \& Zernicke, 1998). These muscles partially compensate the knee ACL ligaments functions: they 
restrict the anterior movement of a tibia relative to a femur and provide resistance to valgus and varus deformations of the leg in the knee joint and to rotation of the tibia (Campbell \& Wayne, 1979). For example, after thigh muscles fatigue in females, $\mathrm{H}$ reflex latency was enhanced and a reduction of reflex responses is associated with increased tibial translation in sagittal plane and ACL injury risk (Behrens et al., 2013). The ECC H strength is decreased for sprinters with a history of the hamstrings injury (Alexander, 1990) and therefore low "functional" H/ Q torque ratios allow to predict the muscle group re-injury. The knee muscles strength and power balance can be altered due to faster fatigue of $\mathrm{H}$ muscles in comparison with $\mathrm{Q}$ muscle observed also in amateur male handball players during isokinetic exercises at the velocity of $240 \% \mathrm{~s}$ (Pontaga \& Zidens, 2015). After an isokinetic fatigue protocol of the Q and $\mathrm{H}$ muscles, female middle distance runners contacted the ground with a greater knee-flexion angle (Kellis et al., 2011), which was accompanied by an antagonist-inhibition strategy around the knee. The ECC maximal $\mathrm{H}$ torque (both thighs) was reduced in long distance runners 18 hours after a marathon (Koller et al., 2006). ECC $\mathrm{H}$ fatigue may be a risk factor for knee and soft tissue injuries during running.

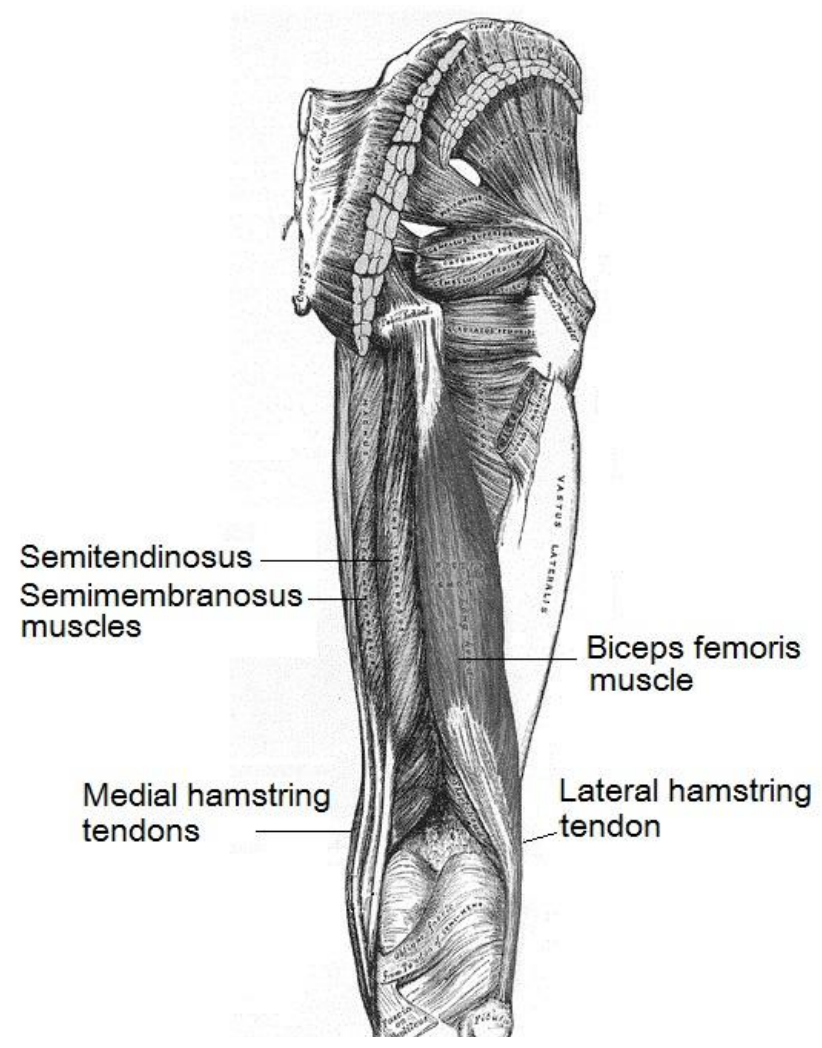

Fig. 1 Posterior group of thigh muscles (hamstrings) (Gray, 1918) 


\section{Material and Methods}

Fifteen amateur female athletes trained in short and middle distances running (students of the Latvian Academy of Sports Education, they had trainings three times per week, mean duration of training was two hours, training experience varied from four to ten years) were informed of the possible test risks and voluntary participated in the investigation. The study was performed in conformity with the standards of the ethics committee of Latvian Council of Sciences. All knee joints were injury - free and painless during the movements. The mean age of the female athletes was $20.5 \pm 1.6$ years, the mean weight 60.3 $\pm 6.3 \mathrm{~kg}$ and the height $1.67 \pm 0.05 \mathrm{~m}$.

The tests were performed by a dynamometer system "REV - 9000" (Technogym, Gambettola, Italy) by isokinetic knee flexion - extension movements. The measurements were corrected for effects of gravity. The range of movements (ROM) in the knee joint was from $10^{\circ}$ in extension to $90^{\circ}$ in flexion (position $0^{\circ}$ of the ROM coincides with full extension of the knee). The person was placed in the positioning seat with the hip at an angle of $115^{\circ}$ of flexion, Fig. 2. (Pontaga, 2004). The hip and trunk were fixed by stabilizing straps. The support lever was attached at the point between the upper two thirds and the lower third of the shin. The person was fixed in position after adjustment of the depth of the seat, the height of the dynamometer and the length of support lever to be aligned with a prolonged virtual rotation axis of the knee. The rotation axis of the knee joint was determined as a line passing through the femoral condyles. The test began with the knee extension from $90^{\circ}$ of the $\mathrm{ROM}$ - extreme flexion position. The movements were repeated five times.

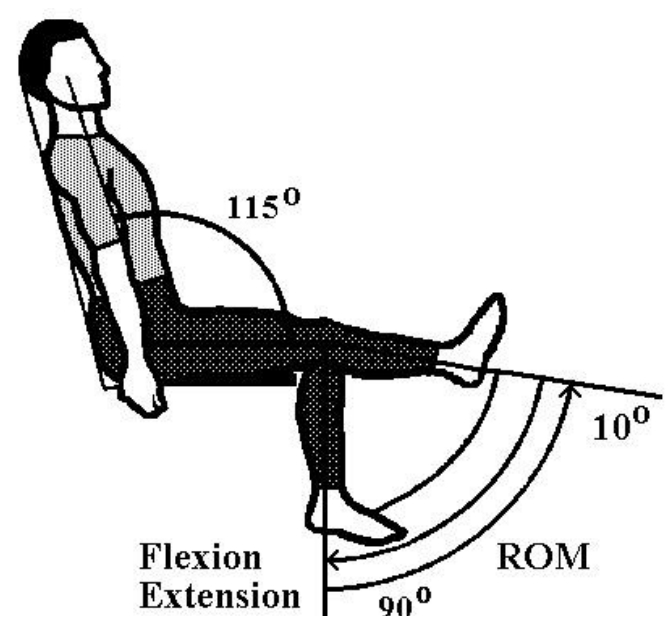

Fig. 2 Range of movements (ROM) in the knee flexion and extension, the positioning seat with the hip angle $115^{\circ}$ of flexion during the isokinetic dynamometry test

(Pontaga, 2004) 
The knee muscles were tested at the angular velocity of $90^{\circ} / \mathrm{s}$ (degrees per second) and $240^{\circ} / \mathrm{s}$ in the in $\mathrm{CC} \mathrm{H} / \mathrm{CC} \mathrm{Q}$ contractions and only at the velocity of $90^{\circ} / \mathrm{s}$ in the ECC H/ CC Q muscles contractions. The torque values produced by $\mathrm{H}$ and $\mathrm{Q}$ muscles are detected at the different angular positions of the ROM of $20^{\circ}, 30^{\circ}, 40^{\circ}, 50^{\circ}, 60^{\circ}, 70^{\circ}, 80^{\circ}$ and $90^{\circ}$ in solely CC contractions and in the ECC $\mathrm{H} / \mathrm{CC} \mathrm{Q}$ muscles contractions.

Just before the investigations and between the tests at different angular velocities passive knee flexion - extension motions at the angular velocity $120 \% \mathrm{~s}$ were performed during 90 seconds. The athletes familiarized themselves with the dynamometric system by a submaximal force of muscles contractions.

The values of maximal torque (measured in $\mathrm{N} \cdot \mathrm{m}$ ) and torque at different angles of the knee flexion and extension movements were obtained from the best repetition at each angular velocity (with the highest maximal torque reached). The results were compared for the CC contractions of both muscles groups and the ECC H/ CC Q muscles contractions. In extreme positions of the joint flexion and extension movements the reliability of the measurements of knee muscles produced torque values was low due to fast changes of the velocity of movements (the movement was not isokinetic). Due to it these values were taken into account only in the middle part of the ROM. The ratios of $\mathrm{H} / \mathrm{Q}$ muscles torques are calculated for the knee joint angles of $30^{\circ}, 40^{\circ}, 50^{\circ}, 60^{\circ}, 70^{\circ}$ and $80^{\circ}$.

Mean values and standard deviations for all characteristics at the velocity of movement of $90 \%$ and $240 \%$ sfor solely CC muscles contractions and at the velocity of $90^{\circ} / \mathrm{s}$ for the ECC H/ CC Q muscles contractions were calculated. Student's $t$ - test for paired data groups was employed to determine differences between characteristics of the CC H/ CC Q muscles and ECC H/ CC Q muscles contractions at the velocity of $90 \%$ and between these characteristics in $\mathrm{CC}$ contractions performed at the velocity of movement of $90 \%$ and $240 \%$. The differences were considered to be statistically significant at $\mathrm{p}<0.05$.

\section{Results}

The mean $\mathrm{H} / \mathrm{Q}$ muscles maximal torques ratio for the female short and middle distances runners in $\mathrm{CC}$ of the both muscles groups at the angular velocity of $90 \%$ is $0.51 \pm 0.07$, but in the ECC H/ CC Q muscles contractions this ratio is $0.60 \pm 0.09$. The difference between the maximal torques ratio in $\mathrm{CC} / \mathrm{CC}$ and $\mathrm{ECC} / \mathrm{CC}$ contractions is significant $(18 \%, \mathrm{p}<0.001)$. This maximal torques ratio in the CC contractions at the fast angular velocity of $240 \% \mathrm{~s}$ is 0.59 \pm 0.09 . The difference between the CC/CC contractions in medium $\left(90^{\circ} / \mathrm{s}\right)$ and fast $\left(240^{\circ} / \mathrm{s}\right)$ velocity of motions is significant $(16 \%, \mathrm{p}<0.001)$. 
The angular H/ Q muscles torques ratios are significantly higher at fast velocity of movement of $240^{\circ}$ /sin comparison with the medium velocity of $90^{\circ} /$ sin $\mathrm{CC}$ of the muscles in flexed knee positions $\left(60^{\circ}, 70^{\circ}, 80^{\circ}\right), \mathrm{p}<0.05$. These angular torques ratios do not differ significantly in extended knee positions $\left(30^{\circ}, 40^{\circ}, 50^{\circ}\right)$, Fig. 3 .

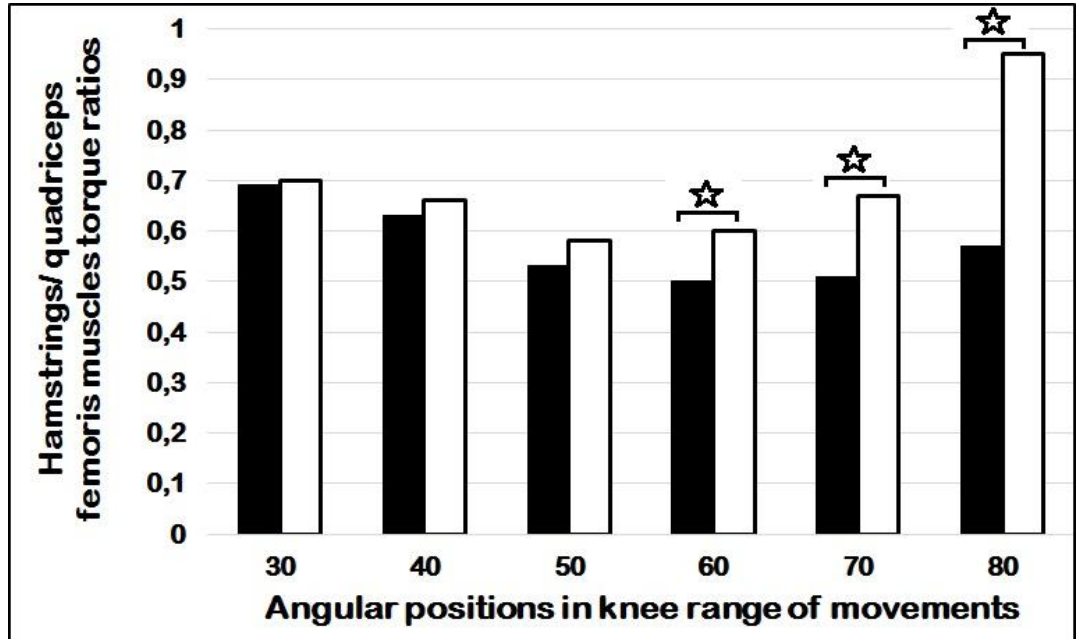

Fig. 3 Hamstrings/ quadriceps femoris muscles torques ratio at the different positions of the ROM at the angular velocity of movement of $90 \% \mathrm{~s}(\square)$ and $240 \% \mathrm{~s}(\square)$ in the concentric contractions for female athletes, $\square$ - difference between the ratios is significant, $\mathrm{p}<0.05$

The angular H/ Q muscles torques ratios are higher for the ECC H/ CC Q muscles contractions than for the $\mathrm{CC}$ of both muscles groups. The differences are significant in the knee extension $\left(30^{\circ}\right)$ and in flexion $\left(70^{\circ}, 80^{\circ}\right)$ angular positions of the ROM, p < 0.05, Fig. 4 .

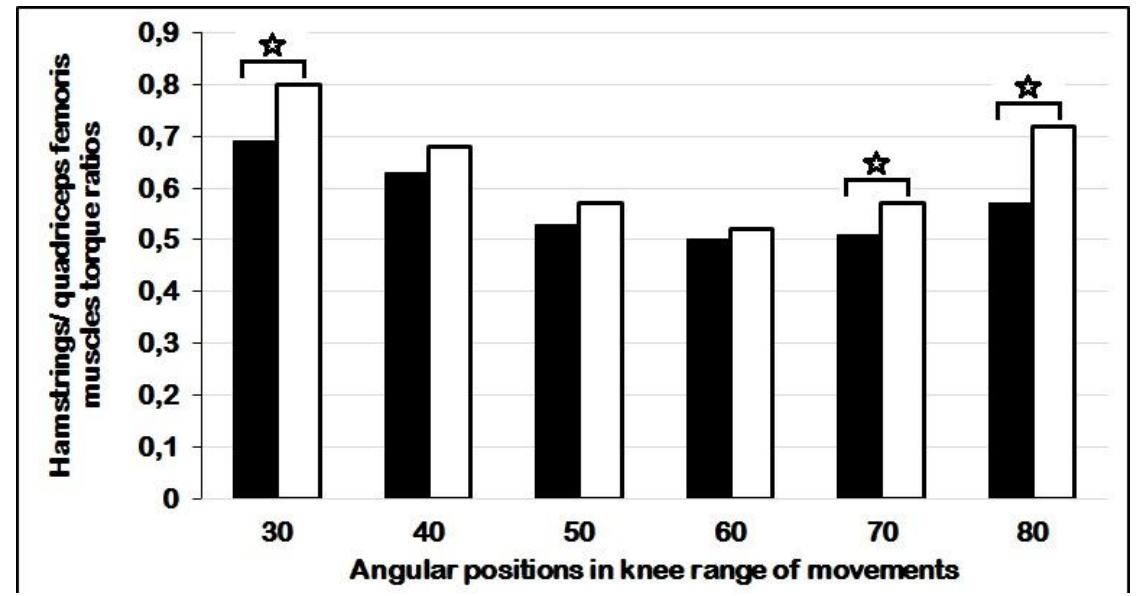

Fig. 4 Hamstrings/ quadriceps femoris muscles torques ratio at the different positions of the ROM at the angular velocity of movement of $90^{\circ} / \mathrm{s}$ in the concentric/ concentric ( $(-)$ and eccentric/ concentric $(\square)$ contractions for female athletes, $\square$ - difference between the ratios is significant, $\mathrm{p}<0.05$ 


\section{Discussion}

To estimate the balance or imbalance in the joint it is necessary to determine flexors/ extensors strength ratios for these two muscles' groups. An imbalance of muscles may cause the weaker muscle group to lie more vulnerable to stress (Osternig, 1986). The mean value for the knee flexors/ extensors (H/ Q) torques ratio is approximately 0.60 at slow angular velocity of movement $30^{\circ}-60^{\circ} / \mathrm{s}$

(Calmels et al., 1997; Kellis \& Baltzopoulos, 1995; Nosse, 1982). This value of this ratio increases with the growth of the velocity of movement, and the value is close to 0.80 at the fast velocity $240^{\circ}-300^{\circ} / \mathrm{s}$ in males (Alexander, 1990; Francis \& Hoobler, 1987; Osternig, 1986). In previous investigation we determined that the $\mathrm{H} / \mathrm{Q}$ torques ratio at the medium angular velocity of $100 \% \mathrm{~s}$ is $0.61 \pm 0.07$, but at the high velocity $200 \% \mathrm{~s}: 0.70 \pm 0.09$ in male basketball and handball players (Pontaga, 2004). From review data of Hewett et al., (2008) the relative maximal $\mathrm{H}$ strength does not change as the velocity increased in females ( $\mathrm{H} / \mathrm{Q}$ ratio is $0.49 \pm 0.09$ at $30^{\circ} / \mathrm{s} ; 0.51 \pm 0.06$ at $\left.360^{\circ} / \mathrm{s}\right)$. Females, unlike males, do not increase $\mathrm{H} / \mathrm{Q}$ torque ratios at velocities that approach those of functional activities. Our results obtained on female sprint and middle distance runners $(\mathrm{H} / \mathrm{Q}$ is $0.51 \pm 0.07$ at $90 \%$ s; $0.59 \pm 0.09$ at $240 \%$; $;<0.001)$ do not support their findings. This can be explained by sport specific adaptation of female thigh muscles to short distance running. Nevertheless H/Q torques ratio in our females at fast motions $(240 \%$ s) is lower than in males: 0.80 (Alexander, 1990; Francis \& Hoobler, 1987; Osternig, 1986).

Changes of the normal H/Q muscles strength ratio puts extra stress on the intra - articular ligaments of the knee, and the ability to restore the correct body alignment in response to sudden external forces is decreased. Therefore the knee joint trauma may occur more probable in sport activities. For example, too high strength of $\mathrm{Q}$ muscle in comparison with $\mathrm{H}$ can be a reason of knee anterior cruciate ligament rupture in athletes (Kannus \& Jarvinen, 1990). The role of the $\mathrm{H}$ muscles is especially important for patients after ACL injury, because these muscles partially compensate the ligaments functions: they restrict the anterior movement of a tibia relative to a femur and provide resistance to valgus and varus deformations of the leg in the knee joint and to rotation of the tibia (Whiting \& Zernicke, 1998). Therefore, ACL rupture with conservative treatment and after reconstruction operation can be a reason of the $\mathrm{H}$ muscles compensatory hypertrophy, and the Q muscle weakness (Lewek et al., 2002; Pontaga \& Larins, 2006). Exercises that use a Q-dominant activation might negatively affect the knee by increasing strain on the anterior cruciate ligament (Begalle et al., 2012). The $\mathrm{H}$ muscles can counteract the deleterious effect of the $\mathrm{Q}$ except when activation is minimal. Exercises with a more balanced Q and H 
coactivation ratio may benefit ACL rehabilitation and knee injury-prevention programs. For example, the most balanced Q-to-H coactivation ratios were produced during the single-limb dead-lift, lateral-hop, transverse-hop and lateral band-walk exercises.

Our opinion coincides with Coombs \& Garbutt (2002) and Nosse (1982), who think that it is not correct to determine the conventional CCratio of $\mathrm{H} / \mathrm{Q}$ for the maximal torques values because they appear in the different positions (angles) of the knee joint range of movements (ROM) and the type of muscles contraction (ECC in some parts of ROM) in real action is not taken into account.

Therefore, we calculated the H/Q torque ratios for the knee joints in different positions of the joints range of movements with the step $10^{\circ}$ and determined that this ratio changes in dependence on the joint angle. This approach allowed us to determine the parts of the ROM, where the certain muscle groups are most vulnerable. Alteration of the strength of muscle groups especially in these parts of the ROM may cause an injury. For $\mathrm{H}$ the maximal strain in muscles appears in the extreme flexion of the hip joint simultaneously with the extreme knee extension. The weakness of extensors in the hip extreme flexion positions (late swing phase) and the extreme knee extension may cause injury of the $\mathrm{H}$ muscles (Whiting \& Zernicke, 1998). The H/Q muscles torques ratio in extended knee positions $\left(30^{\circ}, 40^{\circ}\right.$ and $\left.50^{\circ}\right)$ are similar in medium $\left(90^{\circ} / \mathrm{s}\right)$ and fast $\left(240^{\circ} / \mathrm{s}\right)$ velocity of motions (Fig. 3.). This proves that concentric action of $\mathrm{H}$ cannot prevent extreme knee extension and possible injuries of intraarticular ligaments and $\mathrm{H}$ sprain. This ratio in flexed knee positions $\left(60^{\circ}, 70^{\circ}\right.$ and $\left.80^{\circ}\right)$ is significantly higher at fast $\left(240^{\circ} / \mathrm{s}\right)$ velocity of movement in comparison with medium $\left(90^{\circ} / \mathrm{s}\right)$. This can prevent sprain of $\mathrm{H}$ in the hip extreme flexion positions during sprint running. Our data proves that $\mathrm{ECC}$ action of $\mathrm{H}$ can to decelerate the knee extension to prevent the injury of knee intra - articular ligaments (Whiting \& Zernicke, 1998) because the angular H/Q muscles torques ratios are higher for the $\mathrm{ECC} \mathrm{H/} \mathrm{CC} \mathrm{Q} \mathrm{muscles} \mathrm{contractions} \mathrm{than} \mathrm{for} \mathrm{the} \mathrm{CC}$ in the knee extension $\left(30^{\circ}\right)$ and in flexion $\left(70^{\circ}, 80^{\circ}\right)$ angular positions of the ROM, p $<0.05$, Fig. 4.

Aagaard et al., (1997; 1998) investigated "functional" H/Q muscles strength ratios as ECC $\mathrm{H}$ and $\mathrm{CC} \mathrm{Q}$ contraction in the knee extension, and $\mathrm{CC} \mathrm{H}$ and ECC Q contraction in the knee flexion. Aagaard et al., (1997) determined this "functional" $\mathrm{H} / \mathrm{Q}$ ratio at the angle of ROM $50^{\circ}\left(0^{\circ}\right.$ - full knee extension): it was $0.80-1.00$ for elite sailors and $0.80-0.84$ for male controls at the fast movements $\left(240^{\circ} / \mathrm{s}\right)$. The conventional H/QCC maximal torques ratios were close to 0.50 . In our investigation the conventional H/QCC maximal torques ratio is very close to Aagaard et al., (1997) finding: $0.53 \pm 0.11$, but the ECC H/ $\mathrm{CC} Q$ torques ratio at the angle of ROM $50^{\circ}$ is only $0.57 \pm 0.11$ for female sprint and middle distance runners (see Fig. 4.). This can be explained by using of 
lower angular velocity of movement in the test $\left(90^{\circ} / \mathrm{s}\right)$ and by lower response of $\mathrm{H} / \mathrm{Q}$ torques ratio on velocity of motions and contraction mode in females in comparison with males (Hewett et al., 2008).

Aagaard et al., (1998) determined that the ECC H/ CC Q muscle torques ratios increased during the fast knee extension in the ROM angles $50^{\circ}, 40^{\circ}, 30^{\circ}$. This proves the role of $\mathrm{H}$ in the knee joint stability providing at the fast movements $\left(240^{\circ} / \mathrm{s}\right)$. Investigations of Coombs \& Garbutt (2002) also has shown that there is a continual rise in the ECC H/ CC Q ratio when extending the leg compared to the relatively unchanged $\mathrm{CC} \mathrm{H/CC} \mathrm{Q} \mathrm{ratio.} \mathrm{From} \mathrm{our} \mathrm{results} \mathrm{only}$ ECC H/ CC Q muscles torques ratios significantly increase during the knee extension in the ROM angles from $60^{\circ}$ to $30^{\circ}$ (see Figs. 3.and 4.). This is in good agreement with the observations of Aagaard et al., (1998) and Coombs \& Garbutt (2002). The ratio of the ECC H/ CC Q muscles torques is significantly higher than in solely CC contractions only in the extended knee positions $\left(30^{\circ}\right)$ (Fig. 4.) and flexed knee positions $\left(70^{\circ}\right.$ and $\left.80^{\circ}\right)$ for females. This can be explained with the assumption that the contraction of $\mathrm{H}$ muscles in daily and sport movements is ECC only in the extreme positions of the range of movements (knee extension and flexion), but this contraction is concentric in the middle part of the ROM.

Using is okinetic dynamometers the contractions of muscles can be $\mathrm{CC}$ or ECC in full range of movements. They are "unnatural", because during the real sport activities the ECC muscles action occurs only through few degrees of movement of the ROM.

The balance of the H/Q torques ratio changes dynamically in the range of movements. Therefore, we suggest that it is necessary to determine the alteration of this ratio in the different angles of $\mathrm{ROM}$ in $\mathrm{ECC} / \mathrm{CC}$ and $\mathrm{CC} / \mathrm{CC}$ contractions of the muscles. This will allow to detect the parts of the ROM, where the $\mathrm{H}$ muscles are weak and more probable may be traumatized. Therefore it will give possibility to elaborate exercises for the $\mathrm{H}$ strength and power training in the proper part of the ROM.

\section{Conclusions}

The knee flexor/ extensor muscles maximal torques ratio in eccentric hamstring/ concentric quadriceps femoris muscles contractions at the angular velocity of $90^{\circ} / \mathrm{s}: 0.60 \pm 0.09$ and at the fast concentric contractions of $240^{\circ} / \mathrm{s}$ : $0.59 \pm 0.09$ - both are significantly higher in comparison with this ratio in concentric contractions of muscles at the velocity of $90^{\circ} / \mathrm{s}: 0.51 \pm 0.13(\mathrm{p}<$ $0.05)$.

The hamstrings/ quadriceps muscles torques ratio in only concentric contractions in extended knee positions $\left(30^{\circ}, 40^{\circ}\right.$ and $\left.50^{\circ}\right)$ are similar in medium 
$\left(90^{\circ} / \mathrm{s}\right)$ and fast $\left(240^{\circ} / \mathrm{s}\right)$ velocity of motions, but in flexed knee positions $\left(60^{\circ}\right.$, $70^{\circ}$ and $\left.80^{\circ}\right)$ this ratio is significantly higher in fast movements $(\mathrm{p}<0.05)$.

The hamstring/ quadriceps muscles torques ratio in the knee extreme extension $\left(30^{\circ}\right)$ and flexion $\left(70^{\circ}, 80^{\circ}\right)$ at the eccentric contraction of hamstrings is significantly higher due to greater torques produced by the hamstrings in comparison with quadriceps muscle $(\mathrm{p}<0.05)$.

\section{References}

Aagaard, P., Simonsen, E. B., Beyer, N., Larsson, B., Magnusson, P., \& Kjaer, M. (1997). Isokinetic muscle strength and capacity for muscular knee joint stabilization in elite sailors. International Journal of Sports Medicine, 18. 521-525. PMID: 9414075.

Aagaard, P., Simonsen, E. B., Magnusson, P., Larsson, B., \& Dyhre-Poulsen, P. (1998). A new concept for isokinetic hamstring: quadriceps muscle strength ratio. American Journal of Sports Medicine, 26, 231-237. DOI 10.3233/IES-2011-0406.

Alexander, M. J. L. (1990). Peak torque values for antagonist muscle groups and concentric and eccentric contraction types for elite sprinters. Archives of Physical Medicine and Rehabilitation, 71, 334-339. PMID: 2327888.

Begalle, R. L., DiStefano, L. J., Blackburn, T., \& Padua, D. A. (2012). Quadriceps and hamstrings coactivation during common therapeutic exercises. Journal of Athletic Training, 47 (4), 396-405. DOI: 10.4085/1062-6050-47.4.01.

Behrens, M., Mau-Moeller, A., Wassermann, F., \& Bruhn, S. (2013). Effect of fatigue on hamstring reflex responses and posterior-anterior tibial translation in men and women. PLOS ONE, 8 (2), e56988. Downloaded from: www.plosone.org

Calmels, P. M., Nellen, M., Borne, van der I., Jourdin, P, \& Minaire, P. (1997). Concentric and eccentric isokinetic assessment of flexor - extensor torque ratios at the hip, knee and ankle in a sample population of healthy subjects. Archives of Physical Medicine and Rehabilitation, 78, 1224-1230. PMID: 9365353.

Campbell, D. E., \& Wayne, G. (1979). Foot-pounds of torque of the normal knee and the rehabilitated post meniscectomy knee. Physical Therapy, 59, 418-421.

Coombs, R., \& Garbutt, G. (2002). Developments in the use of the hamstring/ quadriceps ratio for the assessment of muscle balance. Journal of Sports Science and Medicine, 1, 56-62. Downloaded from http://www.jssm.org

Francis, K., \& Hoobler, T. (1987). Comparison of peak torque values of the knee flexor and extensor muscle groups using Cybex II and Lido 2,0 isokinetic dynamometers. Journal of Orthopedics and Sports Physical Therapy, 8, 480-483.

Gray, H. (1918). The muscles and fasciae of the thigh. In: Anatomy of the Human Body. Downloaded from http://www.bartleby.com/107/illus434.html

Hewett, T. E., Myer, G. D., \& Zazulak, B. T. (2008). Hamstrings to quadriceps peak torque ratios diverge between sexes with increasing isokinetic angular velocity. Journal of Science and Medicine in Sport, 11, 452-459. DOI: 10.1016/j.jsams.2007.04.009.

Kannus, P., \& Jarvinen, M. (1990). Knee flexor/ extensor strength ratio in follow - up of acute knee distorsion injuries. Archives of Physical Medicine and Rehabilitation, 71, 38-41. PMID: 2297308.

Kellis, E., \& Baltzopoulos, V. (1995). Isokinetic eccentric exercise. Sports Medicine, 19, 202 -222. PMID: 7784759. 
Kellis, E., Zafeiridis, A., \& Amiridis, I. G. (2011). Muscle coactivation before and after the impact phase of running following isokinetic fatigue. Journal of Athletic Training, 46 (1), 11 -19. DOI: 10.4085/1062-6050-46.1.11.

Knapik, J. J., Bauman, C. L., Jones, B. H., Harris, J. M., \& Vaughan, L. (1991). Preseason strength and flexibility imbalances associated with athletic injuries in female collegiate athletes. American Journal of Sports Medicine, 19, 76-80. DOI: 10.1177/036354659101900113.

Koller, A., Sumann, G., Schobersberger, W., Hoertnagl, H., \& Haid, C. (2006). Decrease in eccentric hamstring strength in runners in the Tirol Speed Marathon. British Journal of Sports Medicine, 40, 850-852. DOI: 10.1136/bjsm.2006.028175.

Lewek, M., Rudolph, K., Axe, M., \& Snyder-Mackler, L. (2002). The effect of insufficient quadriceps strength on gait after anterior cruciate ligament reconstruction. Clinical Biomechanics, 17, 56-63. DOI: 10.1016/S0268-0033(01)00097-3.

Marshall, J. L., \& Tischler, H. M. (1978). Screening for sports: Guidelines. New York State Journal of Medicine, 78, 243-251.

Nosse, L. J. (1982). Assessment of selected reports on the strength relationship of the knee musculature .Journal of Orthopedics in Sports Physical Therapy, 4, 78-85.

Osternig, L. R. (1986). Isokinetic dynamometry: implications for muscle testing and rehabilitation. Exercises and Sport Science Review, 14, 45-80. DOI: 10.1249/00003677-198600140-00005.

Pontaga, I. (2004). Hip and knee flexors and extensors balance in dependence on the velocity of movement. Biology of Sport (Warsaw, Poland), 21, 261-272. ICID: 891846.

Pontaga, I., \& Larins, V. (2006). Thigh muscles functional condition of sportsmen after knee anterior cruciate ligament injury and operation. Proceedings of the Latvian Academy of Sciences, 60, 190-193.

Pontaga, I., \& Zidens, J. (2015). Fatigue resistance of thigh muscles in sport games players. In: Kalina RM (ed.) Proceedings of the 1st World Congress on Health and Martial Arts in Interdisciplinary Approach, HMA (pp. 165-169), Czestochowa, Poland. Warsaw: Archives of Budo.

Whiting, W. C., \& Zernicke, R. F. (1998). Biomechanics of Musculoskeletal Injury. U.S.A.: Human Kinetics. 